\title{
Failing to ENHANCE science
}

\author{
Michael E Farkouh, Sameer Bansilal and Valentin Fuster
}

The recent publication of the ENHANCE trial has brought into question our preconceived notions about how we lower LDL cholesterol and whether carotid intimal-medial thickening (CIMT) is a valid surrogate marker for clinical events. We don't believe that the results of ENHANCE, whether in favor of combination therapy or not, should change evidencebased prescription patterns of clinicians. Here we question the statistical power of the trial, emphasizing the need for caution before forming a judgment about ezetimibe.

It is important to compare patients from previous CIMT trials with the ENHANCE population. The patients enrolled in ENHANCE had familial hypercholesterolemia and were treated with a longer duration of background lipid-lowering therapy than patients in previous studies. The ENHANCE patients also had significantly lower baseline CIMT $(\sim 0.70 \mathrm{~mm})$ than patients enrolled in most other lipid-lowering trials $(0.9-1.1 \mathrm{~mm})$. In an era in which medical therapy is more intensive than ever before, it is conceivable that the trial was underpowered to detect a small difference of only $0.05 \mathrm{~mm}$ in the surrogate marker. We may have been asking the carotid-imaging technology to perform the impossible.

The validity of CIMT as a surrogate for clinical outcomes in patients at risk of cardiovascular disease is now under threat. Well-designed trials of therapies for atherosclerosis, such as high-dose statins, have shown that regression of CIMT or reductions in CIMT progression over time mirror reductions in clinical cardiovascular events. Given the very low plaque burden at baseline, limiting the duration of follow-up in ENHANCE to 2 years may have been a major drawback; studies of intima-media thickness using sensitive modalities such as MRI have taken longer to detect changes.
We don't

believe that

the results of

ENHANCE,

whether

in favor of

combination

therapy or not, should change evidence-

based

prescription

patterns...

ME Farkouh is

the Director of, and $S$ Bansilal is a

Cardiology Fellow

at, the Mount Sinai

Heart Clinical Trials

Unit, Mount Sinai

Cardiovascular

Institute, New York,

NY, USA, and

$V$ Fuster is the Editor-

in-Chief of Nature

Clinical Practice

Cardiovascular

Medicine.

\section{Competing interests}

ME Farkouh has declared an association with Merck.

See the article online for ful details of the relationship.

The other authors declared

no competing interests.

www.nature.com/clinicalpractice doi:10.1038/ncpcardio1276
The combination of simvastatin and ezetimibe in ENHANCE seemed to successfully reduce LDL cholesterol, triglyceride and C-reactive protein levels at 24 months -a favorable effect for combination therapy. In light of the early termination of the JUPITER trial, the pleiotropic effects of statins mediated via C-reactive protein have now been linked to reduced clinical events. It is, therefore, possible that combination therapy with ezetimibe is equivalent to maximizing statin monotherapy with more potent compounds such as atorvastatin or rosuvastatin. However, the torcetrapib trials illustrate how favorable changes in a blood marker (HDL cholesterol) do not necessarily lead to beneficial changes in the imaging marker (CIMT) or an improvement in clinical events. The lowering of LDL by a mechanism unrelated to statins may not provide similar clinical benefits. This theory does not nullify the lipid hypothesis, rather it indicates that the mechanism by which lipid targets are achieved could be pivotal in reducing cardiovascular events.

We believe that the most important conclusion from this controversy is that definitive outcome trials of newer therapies are required before therapies are adopted into practice. Practitioners have a responsibility to provide their patients with the best proven therapy. With overwhelming evidence supporting statin therapy to achieve LDL-cholesterol targets and the lack of outcome data for simvastatinezetimibe therapy, ezetimibe-based therapy should be reserved only for those intolerant to statins, those not reaching lipid targets with other proven therapies, or for those patients enrolled in definitive trials such as IMPROVE-IT. In the meantime, let's not make the mistake of giving too much credence to a trial that was underpowered to test the original hypothesis. 